\title{
Arbor
}

\section{Planck, Einstein y los orígenes de la física cuántica}

\section{José Manuel Sánchez Ron}

Arbor CLXVII, 659-660 (Noviembre-Diciembre 2000), 423-436 pp.

La física cuántica nació en diciembre de 1900, cuando Max Planck, hasta entonces un no demasiado conocido fisico teórico de Berlín, se vio obligado a introducir, para explicar la ley de radiación de un cuerpo negro que él mismo había obtenido poco antes, un tipo de discontinuidad de la energía, asociada a una nueva ley fisica: $E=h \cdot v$. En el presente artículo se explica el camino que condujo a Planck a semejante hallazgo, al igual que los problemas que éste planteaba a su descubridor. Fue Albert Einstein, cuyas aportaciones también se consideran, quien en 1905 avanzó por la senda abierta por Planck, insertando de manera mucho más radical la cuantización energética en el seno de la física.

Los orígenes de las teorías que forman eso que de una forma no siempre precisa denominamos "física cuántica" son diversos. No podemos, en efecto, olvidar los problemas que plantearon, esto es, el papel que desempeñaron, la espectroscopia o la radiactividad. Pero, por muy cierto que sea esto, de lo que no hay duda es que la cuantización, la discretización energética introducida por Max Planck y Albert Einstein en, respectivamente, 1900 y 1905, constituyen la piedra angular, absolutamente imprescindible, y más característica de esa física cuántica.

\section{Max Planck}

Max Planck nació en 1858, en Kiel, hijo de Wilhelm Johann Julius, profesor de Jurisprudencia, y Emma (Patzig de soltera). El prestigio y 
autoridad que Planck llegaría a alcanzar en la comunidad científica alemana y mundial no responde tanto a sus capacidades científicas como a su diligencia, rectitud y fortaleza de carácter. Aun cuando es difícil determinar qué es el genio, no es demasiado arriesgado señalar que Planck no fue un genio: fue un científico capaz, muy capaz, pero lejos de la categoría de un Einstein, un Bohr o un Rutherford. Sus profesores en el Maximilians-Gymnasium de Munich (a donde su familia se trasladó cuando, en 1867, su padre obtuvo la cátedra de Derecho Civil en la Universidad de la capital bávara) solían situarle cerca de los primeros puestos de la clase, pero nunca en el primero: cuarto de veintiocho en 1868-69, y de ahí en adelante, quinto de treinta y siete, octavo de veintitres, tercero de veintiuno y cuarto de diecinueve. En lo que sí parecía destacar era en el estudio de religión y en conducta, áreas en las que con mucha frecuencia recibía el premio de su clase. Siempre fue un «hombre de orden».

Cuando le llegó el momento de elegir qué carrera universitaria elegir, el joven Max dudó entre música, filología antigua y física. A pesar de que cuando preguntó al físico de Munich Philipp von Jolly, éste le respondió que no le aconsejaba que estudiara física, ya que todo estaba descubierto después del hallazgo de los principios de la termodinámica, y que no quedaban más que algunas lagunas por completar, Planck eligió finalmente seguir la carrera de Física en la Universidad de Munich, estudios que comenzó el semestre de invierno de 1874-75. Encontramos algunas claves que explican esa decisión en una carta que Planck escribió muchos años después, el 14 de diciembre de 1930, a Joseph Strasser: "Yo podría haberme convertido también en un filólogo o en un historiador. Lo que me llevó a las ciencias exactas surgió de circunstancias más bien exteriores: un curso de matemáticas (el del profesor Gustav Bauer) al que asistí, en la universidad, me suscitó una gran satisfacción interior y me abrió nuevos horizontes. El hecho de que terminase pasándome de las matemáticas puras a la física tuvo que ver con mi pasión por las cuestiones relativas a la concepción del mundo; estas cuestiones, sin duda, no podían ser resueltas por las matemáticas".

En 1877, y hasta 1879, continuó sus estudios en Berlín, donde pudo seguir los cursos de tres gigantes de la ciencia: el fisiólogo y físico Hermann von Helmholtz, el físico Gustav Kirchhoff y el matemático Karl Weierstrass. Sus clases, sin embargo, dejaban que desear: Helmholtz, recordaría Planck años después, «no preparaba sus clases; se interrumpía constantemente para buscar en un cuaderno los datos necesarios; por otra parte, cometía constantemente errores de cálculo en la pizarra, y daba la impresión de aburrirse tanto como nosotros en su curso" ${ }^{1}$. Kirchhoff sí preparaba con cuidado sus lecciones: «cada frase estaba en su lu- 


\section{Planck, Einstein y los orígenes de la física cuántica}

gar. No faltaba ninguna palabra, no sobraba nada. Pero daba la impresión de que todo estaba aprendido de memoria, lo que le convertía en seco y monótono. Admirábamos al orador, pero no lo que decía». En semejantes circunstancias, «el único recurso que me permitía satisfacer mi sed de conocimientos era leer las obras que me interesaban; se trataba, bien entendido, de las que se relacionaban con el principio de energía. Fue así cómo descubrí los tratados de Rudolf Clausius, cuya claridad me impresionó profundamente y en los que me sumergí con un entusiasmo creciente. Admiraba especialmente la formulación exacta que dio de los dos principios de la termodinámica [el la de la conservación de la energía y el del crecimiento de la entropía] y la relación existente entre ellos».

Clausius formó, junto a Helmholtz y Kirchhoff, no importa lo poco atractivas que le resultasen las clases de éstos, los pilares sobre los que construyó su saber físico: «Todos mis conocimientos los debo exclusivamente a la lectura de nuestros maestros», escribió en 1948, "entre los cuales rindo tributo sobre todo a los nombres de Hermann von Helmholtz, Rudolf Clausius y Gustav Kirchhoff» ${ }^{2}$.

Unas palabras acerca de Clausius (1822-1888), el principal maestro de Planck. Junto a Kelvin, pero él en mayor medida que el británico, a Clausius se le debe la formulación del segundo principio de la termodinámica, incluso el propio nombre de "entropía", que introdujo en uno de sus artículos, en el titulado «Sobre formas diferentes de las ecuaciones fundamentales de la teoría mecánica del calor y la conveniencia de su aplicación» ${ }^{3}$. Allí, después de escribir la familiar fórmula para la entropía, escribía:

«Buscamos ahora un nombre apropiado para $S$. Al igual que hemos denominado a $U$ el contenido de trabajo del cuerpo, llamaremos a $S$ el contenido de transformación del cuerpo. Sin embargo, he creido que es más conveniente tomar los nombres de magnitudes científicas importantes de lenguajes antiguos para que aparezcan sin cambios en todos los idiomas contemporáneos. En consecuencia, propongo que llamemos a $S$ la entropía del cuerpo, según la palabra griega " $\eta \tau \rho \circ \eta$ ", que significa «transformación». De forma intencionada, he formado la palabra entropia para que sea lo más parecida posible a la palabra energía, puesto que las dos magnitudes que vienen dadas por estos nombres están tan estrechamente relacionadas en sus significados físicos que parecía apropiada una cierta similitud en sus nombres".

Recurriendo también a este trabajo podemos apreciar una de las propiedades que la entropía tenía para Clausius: "La segunda ley en la forma que la he asignado dice que todas las transformaciones que tienen lu- 


\section{José Manuel Sánchez Ron}

gar en la naturaleza se desarrollan en una cierta dirección, que he denominado el sentido positivo... Pueden tener lugar en el sentido opuesto, esto es, el negativo, pero solamente cuando son compensados al mismo tiempo por tranformaciones positivas».

Si me he detenido en recordar estos dos aspectos de la obra de Clausius es porque resulta que en la manera en que Planck llegó a proponer la ley de radiación de un cuerpo negro que ahora lleva su nombre, al igual que en el procedimiento que siguió para introducir los cuantos, la entropía desempeña un papel central. Y la entropía al estilo de Clausius, esto es, como una magnitud que representa un devenir universalmente válido, y no a la manera, estadística, probabilistica, de Boltzmann.

Siguiendo el ejemplo de Clausius, Planck hizo del estudio de la termodinámica el centro principal de su atención cuando se convirtió en un físico profesional. Comenzando con su tesis doctoral, que dedicó (sin recibir ninguna ayuda de sus profesores) al tema del papel de los procesos irreversibles en la definición de entropía, y que presentó en Munich en 1879.

A pesar de sus esfuerzos, los resultados de su disertación atrajeron muy poca atención: en Munich, el famoso químico Adolf von Baeyer, uno de sus examinadores, le expresó claramente su opinión de que la física teórica le parecía superflua; Helmholtz, quien de hecho había sido uno de los padres fundadores de la termodinámica (en 1847 formuló en toda su generalidad el principio de conservación de la energía) seguramente nunca leyó la tesis; Kirchhoff la desaprobó, y el joven Max no pudo entrar en contacto con Clausius, que nunca contestó a sus cartas y al que no pudo ver en Bonn, porque cuando fue a visitarlo no estaba en su casa.

En cualquier caso, y tras presentar en 1880 la correspondiente Habilitación, pudo enseñar, como privatdozent, en Munich. En 1885 fue designado profesor extraordinario (esto es, sin cátedra) de Física en la Universidad de Kiel, sustituyendo a Heinrich Hertz, el discípulo favorito de Helmholtz. Hertz, para quien Kiel estaba preparando convertir el puesto de profesor extraordinario que ocupaba en el de catedrático, aceptó una oferta de Karlsruhe (en donde, en 1888, llevó a cabo su gran aportación a la física: la demostración experimental de la existencia de las ondas electromagnéticas de baja frecuencia, que se deducía de la teoría del electromagnetismo de Maxwell).

En Kiel, la carrera científica de Planck fue avanzando, poco a poco, centrada todavía en el segundo principio de la termodinámica. Después de sólo cuatro años allí le llegó una nueva, magnífica, oportunidad: nada más y nada menos que de la Universidad de Berlín, la universidad de la capital de Prusia, del centro neurálgico del nuevo Imperio alemán, la ciu- 


\section{Planck, Einstein y los orígenes de la física cuántica}

dad que iba camino de convertirse también en una de las capitales del mundo. De nuevo, a quien Berlín realmente quería era a Hertz, pero éste aceptó una oferta de Bonn (algo que, por cierto, muestra que por entonces la posición de Berlín en el contexto de la ciencia germana todavía no era del indiscutible liderazgo que sería no mucho tiempo después). En su lugar, aunque como profesor extraordinario, eligieron a Planck. Tres años después, en 1892, recibió el nombramiento de catedrático. Dos años más tarde, con el apoyo del propio Helmoltz, fue elegido miembro ordinario de la Academia Prusiana de Ciencias. Llegaba a la cumbre de su profesión. En Berlín pasaría el resto de su vida, y en Berlín, en 1900, lograría su gran éxito científico: la introducción de los cuantos de energía.

\section{Albert Einstein}

Dos de los tres nombres admirados por Planck fueron también especialmente apreciados por Einstein: Helmholtz y Kirchhoff. «He estudiado algo de Helmholtz sobre los movimientos atmosféricos... Cada vez admiro más la mente original y libre de Helmhotz», escribía Einstein a primeros de agosto de 1899 a Mileva Maric, quien más tarde se convertiría en su primera esposa, y algo menos de un año después, el 29 de julio de 1900, «Y como además hace mal tiempo, me he refugiado, desesperado, en el Kirchhoff» ${ }^{4}$.

Pero mientras que Planck admiraba, adoraba acaso sería una expresión más adecuada, a Clausius, Einstein tenía como modelo a Ludwig Boltzmann: «El Boltzmann es magnífico. Casi lo he terminado" ${ }^{5}$, escribía a Mileva el 13 (?) de septiembre de 1900, «Es un expositor magistral. Estoy firmemente convencido de la corrección de los principios de la teoría; esto es, estoy convencido de que para el caso de los gases se trata realmente del movimiento de masas puntuales discretas de magnitud finita que se mueven según ciertas condiciones. Boltzmann acentúa con mucha razón que las fuerzas hipotéticas entre las moléculas no son ningún componente esencial de la teoría, puesto que toda la energía es de tipo cinéti$c o^{6}$. Es un paso más en la explicación dinámica de los fenómenos físicos».

Y si repasamos, al igual que antes hice con los de Clausius, los escritos de Boltzmann, nos encontramos con el, ya mencionado, carácter probabilístico de sus ideas sobre la entropía. Me detendré en los dos volúmenes de sus Vorlesungen über Gastheorie (Conferencias sobre teoría de los gases) (1896, 1898), ejemplares de los cuales se encontraron en la biblioteca de Einstein, con algunas anotaciones de él mismo; fue uno de los libros que estudió de joven. 
Pues bien, en el volumen primero podemos leer frases como las siguientes: «El hecho de que en la naturaleza la entropía tienda a un máximo demuestra que para todas las interacciones (difusión, conductividad calorífica, etc.) de los gases reales las moléculas individuales se comportan según las leyes de la probabilidad en sus interacciones, o al menos que un gas real se comporta como el gas de moléculas desordenadas que tenemos en mente. Se encuentra así que la segunda ley es una ley probabilista» ${ }^{7}$. Por si quedarán dudas, Boltzmann abría la segunda parte de su tratado con la siguiente cita de Williard Gibbs: ${ }^{8}$

«La imposibilidad de una disminución no compensada de entropía parece quedar reducida a una improbabilidad ${ }^{9}$.

En las distintas inclinaciones cientifico-filosóficas existentes entre Planck y Einstein se encuentra, de hecho, la raíz de las diferencias que mantuvieron en lo referente al significado de la cuantización.

\section{Planck y Einstein}

Unas diferencias científicas que tenían su correlato en sus respectivas personalidades. Y es que, por poco que uno sepa de ellos, enseguida se hace evidente cuán diferentes fueron sus personalidades en aspectos centrales. Planck, el funcionario germano cuyas más nobles virtudes ensalzó Max Weber; recuerdese si no cómo definió en 1919 Max Weber al funcionariado moderno: ${ }^{10}$ "un conjunto de trabajadores intelectuales, altamente cualificados y especializados mediante una preparación de años, con un honor estamental muy desarrollado en beneficio de la integridad, sin la cual se cerniría sobre nosotros como un destino el peligro de una terrible corrupción y de una brutal incompetencia e incluso estaría amenazado el rendimiento técnico del aparato estatal». Debido a ese honor, Planck no podía rebelarse contra el Estado, por mucho que éste pudiese comportarse en alguna ocasión de manera que él considerarse indigna. Fue, es cierto, el introductor de una revolución científica, pero a duras penas puede ser considerado un revolucionario.

Por el contrario, Einstein, un auténtico revolucionario científico, fue un hombre al que los nacionalismos y los «honores estamentales» querían decir poco (entre enero de 1896, cuando renunció a la nacionalidad alemana, y 1901, cuando logró la ciudadanía suiza, fue un apatrida). Y aun así, ambos, Planck y Einstein se apreciaron extraordinariamente. Planck se esforzó por llevarlo a Berlín, viajó, con Nernst, incluso a Zurich para 
convencerle de que aceptará — como hizo finalmente- la oferta de la capital prusiana. Por su parte, Einstein respetaba a Planck, no sólo, ni siquiera sobre todo, por sus aportaciones científicas, sino por la persona que era, aunque mantuvieran en ocasiones posturas encontradas. Nadie hizo más que Planck por retenerlo en Berlín. En los peores tiempos, en agosto de 1933, desde Princeton, Einstein escribía a Haber, tras haber sabido que éste también se había convertido finalmente en un exiliado: ${ }^{11}$ «Espero que no regresará a Alemania. No merece la pena trabajar para un grupo intelectual formado por hombres que se apoyan en sus estómagos delante de criminales comunes y que incluso simpatizan en algún grado con estos criminales. No me decepcionan, porque nunca tuve ningún respeto o simpatía por ellos, aparte de unas finas personalidades (Planck, 60\% noble, y Laue, $100 \%$ )».

\section{La introducción de los cuantos de energía}

Como ya he apuntado, fue en Berlín, en 1900, cuando Planck introdujo la cuantización energética en la física.

Esa introducción estuvo asociada al descubrimiento de una nueva ley para la distribución de la densidad de energía de radiación de un cuerpo negro (radiación que está en equilibrio con la materia, y que por tanto absorbe y emite la misma cantidad de energía para cualquier longitud de onda), ley que también propuso Planck en 1900. Ahora bien, ¿cómo es que un físico como Planck, formado en el estudio de los escritos de Clausius, y cuyo programa de investigación se centraba en los principios de la termodinámica, y más concretamente en el segundo, el del crecimiento de la entropía, terminó asociando su nombre a un problema como el de la ley de distribución de la energía de un cuerpo negro? La respuesta a esta pregunta no es difícil: Planck no dudaba de la universalidad del crecimiento de la entropía total (es más, la búsqueda de absolutos, de verdades universales, es lo que orientaba -y siempre orientó- sus trabajos científicos), pero quería, no obstante, relacionar esta irreversibilidad con otras leyes también fundamentales. En concreto quería desarrollar una teoría macroscópica basada en la termodinámica y el electromagnetismo, esperando obtener el principio de irreversibilidad, del crecimiento de la entropía, como parte de esa teoría. Y el problema de la radiación del cuerpo negro se prestaba de manera magnífica para semejante propósito. En primer lugar, lo que se tiene en este caso es un proceso de interacción entre ondas electromagnéticas y materia (la cavidad que aloja a las ondas). En segundo lugar, Planck tenía a su disposición el resultado 


\section{José Manuel Sánchez Ron}

obtenido en 1859 por su antiguo profesor, Gustav Kirchhoff, que asegura que la distribución de radiación en equilibrio es independiente del sistema con el que interacciona la radiación. Era obvio, por su sencillez, considerar entonces a la cavidad del cuerpo negro como formada por una colección de osciladores armónicos cargados.

El problema se planteaba, por consiguiente, en términos del estudio de la interacción entre ondas electromagnéticas y osciladores, para tratar de entender así, mediante procesos de difusión, cómo se obtiene el estado de equilibrio para la radiación del cuerpo negro. Planck esperaba que la simetría temporal de partida en las interacciones electromagnéticas desapareciese a lo largo del proceso, generando de esta manera la irreversibilidad contenida en el segundo principio, que quedaría así «explicado» al estudiar la termodinámica (la entropía, por ejemplo) de la radiación.

Que Planck no fuese capaz de desarrollar este programa, aunque en algún momento creyese que lo había conseguido, es algo que no nos interesa demasiado. Lo importante es señalar que sus investigaciones le prepararon para cuando, en octubre de 1900, Heinrich Rubens y Ferdinand Kurlbraum, colegas de Planck en Berlín, llevaron a cabo en el Physikalisch-Technische Reichsanstalt, el laboratorio nacional alemán, experimentos con los que demostraban que para longitudes de onda grandes la hasta entonces aceptada - aunque con reparos - ley de radiación de Wien no era correcta. Planck reaccionó entonces inmediatamente generalizando heurísticamente lo que hasta entonces había hecho. La modificación que introdujo en sus desarrollos le llevó a una nueva ley de distribución de la radiación del cuerpo negro, ley que presentó en la reunión de la Sociedad de Física Alemana que se celebró en Berlín el 19 de octubre de $1900^{12}$. El día siguiente Rubens le informaba que sus cálculos demostraban que la nueva fórmula se ajustaba perfectamente a los resultados experimentales. Casi inesperadamente, como por sorpresa, Planck se encontró con que disponía de una aparentemente correcta ley de distribución para la radiación del cuerpo negro, cuya explicación teórica, sin embargo, ignoraba (la extensión heurística que había realizado no significaba ninguna explicación teórica).

Naturalmente, Planck se dedicó inmediatamente a la tarea de explicar teóricamente esa ley, lográndolo poco después, en diciembre ${ }^{13}$. Fue entonces cuando se vio obligado a suponer que, de alguna manera, la energía involucrada en la radiación de un cuerpo negro estaba cuantizada, no se podía dividir de manera infinita, que no era continua. Fue también entonces cuando escribió la ecuación que codificaba ese hecho, la célebre: $E=h \cdot v$, donde $h$ es una constante, hoy llamada «constante de Planck», y u la frecuencia. 


\section{Planck, Einstein y los orígenes de la física cuántica}

Más de treinta años después, en una carta que escribió el 7 de octubre de 1931 al físico estadounidense Robert Williams Wood, Planck recordó que, «resumido brevemente, se puede describir lo que hice como un acto de desesperación. Por naturaleza soy pacífico y rechazo toda aventura dudosa. Pero por entonces había estado luchando sin éxito durante seis años (desde 1894) con el problema del equilibrio entre radiación y materia y sabía que este problema tenía una importancia fundamental para la física; también conocía la fórmula que expresa la distribución de la energía en los espectros normales. Por consiguiente, había que encontrar, costase lo que costase, una interpretación teórica. Tenía claro que la física clásica no podía ofrecer una solución a este problema, puesto que con ella se llega a que a partir de un cierto momento toda la energía será trasferida de la materia a la radiación. Para evitar esto se necesita una nueva constante que asegure que la energía no se desintegre. Pero la única manera de averiguar cómo se puede hacer esto es partiendo de un punto de vista definido. En mi caso, el punto de partida fue el mantener las dos leyes de la termodinámica. Hay que conservar, me parece, estas dos leyes bajo cualquier circunstancia. Por lo demás, estaba dispuesto a sacrificar cualquiera de mis convicciones anteriores sobre las leyes físicas. Boltzmann había explicado cómo se establece el equilibrio termodinámico mediante un equilibrio estadístico, y si se aplica semejante método al equilibrio entre la materia y la radiación, se encuentra que se puede evitar la continua transformación de energía en radiación suponiendo que la energía está obligada, desde el comienzo, a permanecer agrupada en ciertos cuantos. Esta fue una suposición puramente formal y en realidad no pensé mucho en ella» ${ }^{14}$.

$\mathrm{El}$ "acto de desesperación" al que se refería fue, como él mismo señalaba, adoptar la formulación estadística de la entropía propuesta por Ludwig Boltzmann en 1877: la célebre expresión para la entropía de un sistema, $S=k \cdot \ln W$, donde $k$ es una constante (introducida precisamente por Planck posteriormente y denominada «constante de Boltzmann») y $W$ la probabilidad de que tenga lugar el estado en cuestión. Doblegarse ante semejante planteamiento, aceptar que el crecimiento de la entropía estaba asociado con probabilidades y que, por consiguiente, no era tan universal como él pensaba, que podía ser violado, debió ser doloroso para un físico del talante de Planck, dolor sólo mitigado haciendo de este paso una "suposición puramente formal».

El por qué Planck consideró el recurso a la ley de Boltzmann como una "suposición formal», o, mejor, la naturaleza de sus problemas para aceptar como definitiva la manera en que había introducido los cuantos, ha sido objeto de numerosos estudios. Una forma de entenderlo es utilizando sus propias palabras. Por ejemplo, las que empleó en abril y mayo 


\section{José Manuel Sánchez Ron}

de 1909, cuando dictó un curso de ocho lecciones en la Universidad de Columbia, en Nueva York, que fue publicado en 1910 en alemán bajo el título Acht Vorlesungen über theoretische Physik (Hitzel, Leipzig), y en 1915 en inglés, Eight Lectures on Theoretical Physics. En la sexta conferencia ("Radiación calorífica. Teoría estadística»), además de reconstruir la manera en que había llegado a la fórmula $\mathrm{E}=\mathrm{h} \cdot \mathrm{v}$, comentó las limitaciones que veía en su derivación: ${ }^{15}$

«Incluso si la formula de radiación derivada aquí se ha mostrado válida con respecto a todas las pruebas precendentes, la teoría todavía requeriría de una extensión con respecto a un cierto punto; ya que en ella el significado fisico de la constante universal $h$ permanece sin explicar. Todos los intentos previos de obtener una fórmula de radiación en base a las leyes conocidas de la teoría del electrón, entre los cuales la teoría de J. H. Jeans debe ser considerado el más general y exacto, han conducido a a la conclusión de que $h$ es infinitamente pequeña, de manera que, por tanto, la fórmula de radiación de Rayleigh posee una validez general. Sin embargo, en mi opinión no hay duda de que esta fórmula pierde su validez para ondas cortas, y que los esfuerzos que se ha tomado Jeans para situar la culpa de la contradicción entre teoría y experimento en este último no tienen fundamento.

En consecuencia, sólo resta una conclusión, que todas las teorías del electrón desarrolladas hasta ahora sufren de una incompletitud esencial que exige una modificación, pero cuán profunda debería ser la modificación en la estructura de la teoría es algo sobre lo que todavía existen puntos de vista muy diferentes... J. J. Thomson se inclina hacia el punto de vista más radical, al igual que J. Larmor, A. Einstein y con él I. Stark, que incluso cree que la propagación de las ondas electromagnéticas en el vacío no tiene lugar de acuerdo con las ecuaciones del campo de Maxwell, sino en cuantos de energía definidos, hu. Por el contrario, yo soy de la opinión de que en la actualidad no es necesario proceder de semejante forma revolucionaria, y que se puede salir adelante con éxito buscando el significado del cuanto de energía hu únicamente en las acciones mutuas con las que los osciladores se influyen entre sím.

Y en este punto añadía en una nota a pie de página: «Es mi intención dar una relación completa de estas relaciones en el volumen 31 del $A n$ nalen der Physik».

\section{Einstein sobre Planck}

Gracias a su correspondencia, sabemos que Einstein conocía desde al menos la primavera de 1901 los trabajos de Planck. En efecto, el 4 de 


\section{Planck, Einstein y los orígenes de la física cuántica}

abril de 1901 escribía a Mileva: «Me han surgido objecciones de principio contra los estudios sobre radiación de Planck, de suerte que leo su tratado con sentimientos encontrados». Y una semana después, el 10 de abril: «Lo que me preocupa de las observaciones de Planck sobre la naturaleza de la radiación se dice pronto. Planck supone que una clase muy determinada de resonadores (con períodos y amortiguamientos determinados) ocasiona la conversión de la energía en radiación, un supuesto con el que yo no estoy de acuerdo. Tal vez su última teoría sea más general».

Tenía problemas con los cuantos introducidos por Planck en 1900, pero esto no fue óbice para que en 1905, su annus mirabilis, diera un paso más en la cuantización al proponer, en un artículo titulado «Sobre un punto de vista heurístico acerca de la producción y transformación de la luz», que "las observaciones asociadas con la radiación del cuerpo negro, fluorescencia, producción de rayos catódicos mediante luz ultravioleta y otros fenómenos relacionados, todos ellos conectados con la emisión o transformación de la luz, se entienden más fácilmente si uno supone que la energía de la luz está distribuida espacialmente de forma discontinua ${ }^{16}$. Para llegar a semejante conclusión, para suponer que la luz se comporta también como si estuviese formada por partículas (cuantos, fotones como los llamamos desde que el químico-físico Gilbert Newton Lewis propusiera el término en la década de 1920) independientes, utilizó no la ley de radiación de Planck sino la de Wien, válida únicamente para un extremo del espectro; en base a ella calculó la entropía - de nuevo la entropía-, encontrando que depende del volumen «como si la radiación fuese un medio discontinuo consistente de cuantos de energía independientes".

Mientras que Planck pensaba que su cuantización debía representrar algún mecanismo del intercambio energético entre los osciladores cargados que forman la pared de la radiación, con su cuantización, la segunda, Einstein insertaba la discontinuidad energética en la propia estructura de la radiación, otrora supuesta completamente continua. Ahora bien, era perfectamente consciente no sólo de las limitaciones de su propio trabajo, ya indicadas en el propio título del artículo ("Sobre un punto de vista heurístico...»), sino también de las del de Planck de 1900. Abundan los lugares en donde dejó constancia escrita de su insatisfacción. Uno de esos lugares, particularmente claro, es en su artículo de 1916 titulado «Emisión y absorción de radiación en la teoría cuántica» ${ }^{17}$. Citaré de él:

«Hace dieciseis años, cuando Planck creó la teoría cuántica deduciendo su fórmula de radiación, adoptó la siguiente aproximación. Calculó la energía media $\overline{\mathrm{E}}$ de un resonador como función de la temperatura según sus re- 
cientemente encontrados principios cuánticos teóricos, y determinó a partir de ahí la densidad de radiación $\rho$ como una función de la frecuencia $u$ y temperatura. Logró esto deduciendo - basándose en consideraciones electromagnéticas - una relación entre la densidad de radiación y energía del resonador $\overline{\mathrm{E}}$ :

$$
\overline{\mathrm{E}}=\frac{\mathrm{c}^{3} \rho}{8 \pi \mathrm{u}^{2}}
$$

Su deducción fue de un atrevimiento sin precedentes, pero encontró brillante confirmación. No sólo fue confirmada la propia fórmula de radiación y el valor calculado del cuanto elemental, sino que también fue confirmado por posteriores investigaciones sobre el calor específico el valor calculado recurriendo a la teoría cuántica de $\overline{\mathrm{E}}$. De esta forma, la ecuación (1), hallada inicialmente mediante la teoría electromagnética, también fue confirmada. Sin embargo, continuó siendo insatisfactorio que el análisis mecanico-electromagnético, que condujo a (1), sea incompatible con la teoría cuántica, y no es sorprendente que el propio Planck y todos los teóricos que trabajan en este tema traten incesantemente de modificar la teoría para basarla en cimientos no contradictorios.

Con los grandes éxitos de la teoría espectral de Bohr, parece ya no quedar dudas de que la idea básica de la teoría cuántica debe ser mantenida. Parece, por consiguiente, que debe establecerse la uniformidad de la teoría de tal manera que las consideraciones electromagnéticas, que condujeron a Planck a la ecuación (1), sean reemplazadas por argumentos mecanicocuánticos sobre la interacción entre materia y radiación».

Y puesto que estoy con este artículo, diré que para Einstein constituyó un paso muy importante en el "problema de los cuantos". Veamos, en este sentido, lo que manifestaba a su amigo Michele Angelo Besso en una carta que le escribió el 11 de agosto de 1916, esto es, después de haber completado el artículo (éste fue recibido por la revista el 17 de julio, de 1916, y publicado el 30 de julio): ${ }^{18}$

«He tenido un destello de lucidez a propósito de la absorción y emisión de radiación; esto te interesará. Una demostración completamente sorprendente de la fórmula de Planck, yo incluso diría la demostración. Todo completamente cuántico. Estoy ahora redactando este resultado». ${ }^{19}$

Ciertamente, no fue la demostración; eso es algo que tendría que esperar a 1924, al artículo en el que Satyendra Nath Bose introdujo una nueva estadística (la estadística de Bose-Einstein). Pero aun así Einstein se entusiasmó con su trabajo, que, por otra parte contiene novedades tan importantes como los coeficientes de absorción y emisión espontánea e 


\section{Planck, Einstein y los orígenes de la física cuántica}

inducida. El 24 de agosto de 1916 volvía a mostrar su entusiasmo en otra carta a Besso:

"Te he enviado, hace ya mucho tiempo, mis trabajos sobre gravitación y sobre la fórmula de Planck. El segundo te gustará. ${ }^{20} \mathrm{El}$ desarrollo es puramente cuántico y proporciona la fórmula de Planck. Analizándolo, se puede mostrar de forma convincente que los procesos elementales de la emisión y absorción son procesos dirigidos. Basta examinar el movimiento (browniano) de una molécula (según el desarrollo hallado) en el dominio de la emisión luminosa. Y esta demostración, que aparece en el folleto de la Zürcher Phys. Ges ${ }^{21}$. en homenaje a Kleiner, se hace igualmente sin ningún razonamiento relevante de la teoría ondulatoria».

Y unos días más tarde, el 6 de septiembre, añadía: «Los trabajos de Planck no proporcionan ninguna relación entre $h$ y $\varepsilon$. Se tiene una vaga idea de la homogeneidad de la fórmula y del hecho de que los órdenes de magnitud de $\varepsilon^{2} / \mathrm{c}$ y de $h$ son muy próximos, pero este último punto todavía no ha sido aclarado por ninguna teoría. Para obtener la ley del desplazamiento de Wien se necesita el principio de Doppler y la ley de presión de la radiación que, hasta aquí, no han sido formuladas, como por otra parte la noción de frecuencia, sino por medio de la teoría ondulatoria. Lo que hay de esencial es que las consideraciones estadísticas que conducen a la formula de Planck se han sistematizado, y que se puede concebir la cosa de una manera general por el hecho de que, para la constitución particular de las moléculas consideradas, se ha partido únicamente de la idea más general de los cuantos. Esto conduce al resultado (que todavía no se encuentra en el trabajo que te enviado) de que, cuando existe intercambio de energía elemental entre la radiación y la materia, se transfiere el impulso hu/c a la molécula. Se deduce que todo proceso elemental de esta naturaleza es un proceso enteramente orientado ${ }^{22}$. Así, queda establecida la existencia de los cuantos de luz».

No era, como ya he señalado, estrictamente cierto que Einstein hubiese demostrado «la existencia de los cuantos de luz», no al menos de una forma totalmente satisfactoria, pero aún así, cuánto se había avanzado desde 1900, desde que Planck escribiera aquella fórmula, $E=h \cdot v$, que ya forma, y continuará seguramente formando, parte de la cultura universal.

\section{Notas}

1 M. Planck, Physikalische Abhandlungen und Vorträge (Braunschweig 1958), vol. III, p. 375. 


\section{José Manuel Sánchez Ron}

2 Max Planck in seinen Akademie-Ansprachen (Berlín 1948), p. 4.

3 R. Clausius, “Über verschiedene für die Anwendung bequeme Formen der Hauptgleichungen der mechanischen Wärmetheorie", en Abhandlungen über die mechanische Wärmetheorie, vol. II (Vieweg, Braunschweig 1867), pp. 1-44.

4 Estas cartas aparecen en The Einstein Collected Papers, vol. 1 (The Early Years, 1879-1902), John Stachel, ed. (Princeton University Press, Princeton 1897). Ahí se indica que la obra de Kirchhoff a la que se hace referencia es seguramente: G. Kirchhoff, Vorlesungen über mathematische Physik, vol. 1 (Mechanik), 4a ed., W. Wien, ed. (Teubner, Leipzig 1897). Existe traducción al castellano de las cartas a Mileva que estoy citando: A. Einstein, Cartas a Mileva, José M. Sánchez Ron, ed. (Mondadori, Madrid 1990).

5 Seguramente L. Boltzmann, Vorlesungen über Gastheorie, parte 1, Theorie der Gase mit einatomigen Molekülen, deren Dimensionen gegen die mittlere Weglänge verschwinden (Leipzig, 1896) y Vorlesungen über Gastheorie, parte 2, Theorie van der Waals'; Gase mit zusammengesetzten Molekülen; Gasdissociation; Schlussbe-merkungen (Leipzig, 1898).

6 Ver L. Boltzmann, Vorlesungen über Gastheorie, parte 1, Theorie der Gase mit einatomigen Molekülen, deren Dimensionen gegen die mittlere Weglänge verschwinden, op. cit., p. 15.

7 He utilizado la versión inglesa de Stephen G. Brush: L. Boltzmann, Lectures on Gas Theory (University of California Press, Berkeley 1964), pp. 74-75.

8 Ibid., p. 215.

9 Esta frase aparece en J. W. Gibbs, "On the equilibrium of heterogeneous substances", Transactions of the Connecticut Academy 3, 108-248 (1875); reproducido en The Scientific Papers of J. Williard Gibbs, vol. 1 (Thermodynamics) (Dover, Nueva York 1961); la cita aparece en la p. 167, dentro de la sección titulada "Considerations relating to the increase of entropy due to the mixture of gases by diffusion".

10 M. Weber, La política como profesión (Espasa-Calpe, Madrid 1992), p. 108.

11 Fritz Stern, Einstein's German World (Princeton University Press, Princeton, 1999), pp. 159-160.

12 M. Planck, “Über eine Verbesserung der Wien'schen Spektralgleichung", Verhandlungen der Deutschen Physikalischen Gesellschaft 2, 202-204 (1900).

13 M. Planck, "Zur Theorie des Gesetzes der Energieverteilung im Normalspektrum", Verhandlungen der Deutschen Physikalischen Gesellschaft 2, 237-243 (1900).

14 Citada en Hermann, Armin, The Genesis of Quantum Theory (1899-1913) (The MIT Press, Cambridge, Mass. 1971), pp. 23-24.

15 M. Planck, Eight Lectures on Theoretical Physics (Dover, Nueva York 1998), pp. 95-96.

16 A. Einstein, “Über einen die Arzeugung und Verwandlung des Lichtes betreffenden heuristischen Gesuchtspunkt", Annalen der Physik 17, 132-148 (1905).

17 A. Einstein, "Strahlungs-Emission und -Absorption nach der Quantentheorie", Deutsche Physikalische Gesellschaft. Verhandlungen 18, 318-323 (1916).

18 Albert Einstein, Correspondencia con Michele Besso (Tusquets, Barcelona 1994).

19 De esta época son dos artículos de Einstein referentes a la teoría cuántica: el ya citado (ver nota 17) "Strahlungs-Emission und -Absortion nach der Quantentheorie", y "Quantentheorie der Strahlung", Mitteilungen der Physikalischen Gesselschaft Zürich 16, 47-62 (1916).

20 A. Einstein, «Strahlungs-Emission und -Absorption nach der Quantentheorie», op. cit. nota 17.

21 A. Einstein «Quantentheorie der Strahlung», op. cit. nota 19.

22 A. Einstein "Quantentheorie der Strahlung", Physikalische Zeitschrift 18, 121-128 (1917). 